\title{
Comparison of body-powered voluntary opening and voluntary closing prehensor for activities of daily life
}

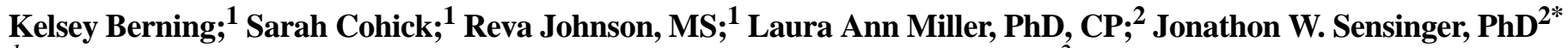 \\ ${ }^{1}$ Department of Biomedical Engineering, Northwestern University, Evanston, IL; ${ }^{2}$ Department of Physical Medicine and \\ Rehabilitation, Feinberg School of Medicine, Northwestern University, Chicago, IL; and Center for Bionic Medicine, \\ Rehabilitation Institute of Chicago, Chicago, IL
}

\begin{abstract}
Persons with an upper-limb amputation who use a body-powered prosthesis typically control the prehensor through contralateral shoulder movement, which is transmitted through a Bowden cable. Increased cable tension either opens or closes the prehensor; when tension is released, some passive element, such as a spring, returns the prehensor to the default state (closed or open). In this study, we used the Southampton Hand Assessment Procedure to examine functional differences between these two types of prehensors in 29 nondisabled subjects (who used a body-powered bypass prosthesis) and 2 persons with unilateral transradial amputations (who used a conventional body-powered device). We also administered a survey to determine whether subjects preferred one prehensor or the other for specific tasks, with a long-term goal of assessing whether a prehensor that could switch between both modes would be advantageous. We found that using the voluntary closing prehensor was $1.3 \mathrm{~s}$ faster $(p=0.02)$ than using the voluntary opening prehensor, across tasks, and that there was consensus among subjects on which types of tasks they preferred to do with each prehensor type. Twenty-five subjects wanted a device that could switch between the two modes in order to perform particular tasks.
\end{abstract}

Key words: amputation, artificial limbs, body-powered prostheses, grasp, outcomes assessment, prehensor, prosthesis design, terminal device, voluntary closing, voluntary opening.

\section{INTRODUCTION}

The majority of persons with an upper-limb amputation use body-powered prostheses [1], which are simple, robust, and inexpensive [2]. Control of body-powered prostheses is achieved through a Bowden cable that couples motion of an intact joint to movement of the terminal device, allowing better control of accuracy [3] and more intuitive feedback [4] than myoelectric prostheses [5-6]. Indeed, body-powered prostheses are the primary device used by 78 percent of Vietnam veterans and 38 percent of Operation Iraqi Freedom/Operation Enduring Freedom veterans with an upper-limb amputation [7]. However, body-powered prostheses are still abandoned at high rates, with estimates ranging between 26 [8] and 50 percent [9]. One of the main causes of abandonment is the limited function of the prehensor [9], especially when compared with an intact contralateral hand.

\footnotetext{
Abbreviations: $\mathrm{ANOVA}=$ analysis of variance, $\mathrm{IOF}=$ index of functionality, SHAP = Southampton Hand Assessment Procedure, $\mathrm{VC}=$ voluntary closing, $\mathrm{VO}=$ voluntary opening.

${ }^{*}$ Address all correspondence to Jonathon W. Sensinger, PhD; Institute of Biomedical Engineering, University of New Brunswick, 25 Dineen Dr, PO Box 4400, Fredericton, NB E3B 5A3; 312-238-6036; fax: 312-238-2081. Email: sensinger@ieee.org http://dx.doi.org/10.1682/JRRD.2013.05.0123
} 
In contrast to the complex movements possible in an intact human hand, body-powered terminal devices typically only allow control of a single hinge joint. In addition, they can only control one direction of joint movement. Both human muscles and Bowden cables only generate power when in tension; however, humans use at least two muscles to control each joint, thus, for example, allowing both opening and closing of the hand to be actively controlled. In contrast, to maintain device simplicity, body-powered prostheses use a single Bowden cable. A choice must accordingly be made on how the active force generated by the user and transferred via the cable should be used, i.e., whether the cable force should open or close the prehensor. In a voluntary closing (VC) device, the user actively closes the device and some passive element, such as a spring, returns the prehensor to the default open state. In a voluntary opening (VO) device, the user actively opens the prehensor and a passive spring restores the default closed state.

Most body-powered split-hook prehensors used are VO devices (e.g., Figure 1(a)), in which a passive spring closes the device and hence determines the grasp force. An advantage of VO prehensors is that, after actively opening the fingers and positioning them around an object, the user can relax because the spring provides the grip force to hold the object. This works well for the majority of moderately weighted objects encountered in daily tasks. However, the VO prehensor cannot generate more force than that supplied by the spring. The user is thus forced to select a spring strength based on the maximum grip force they anticipate needing; a stronger spring is required for heavy, irregularly shaped, or slippery objects. This has two implications. First, it means that the user must overcome a larger than necessary spring force to open the device when manipulating lightweight objects that only require a low pinch force-likely the majority of objects encountered-and thus typically expend unnecessary energy. Second, it means the user cannot properly manipulate objects that, whether due to excessive weight, awkward shape, or slippery surface, require more grip force than the spring provides. Some VO prehensors allow the user to adjust spring tension [10-11], but only over a limited range of forces. Thus, while VO prehensors seem suitable for many tasks, they do not work well in an important subset of activities.

VC devices (e.g., Figure 1(b)), in which a spring is used to overcome friction and return the finger to the open state, are often used by children, and by some adults

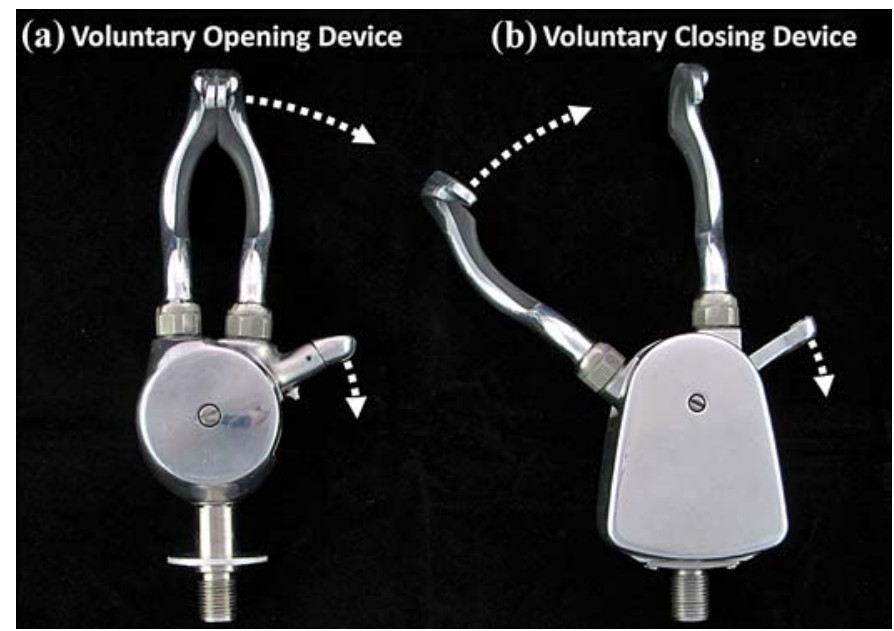

Figure 1.

Voluntary opening (VO) vs voluntary closing (VC) terminal devices. Cable of body-powered prostheses pulls lateral leverarm proximally (downward in picture). Depending on device, this movement can either pull finger open ((a) VO mode) or push the finger closed ((b) VC mode). Spring returns device to its default state when user releases tension on cable. VO device used in study was Sierra 2-load hook (with custom extension added to make it same length as VC device). VC device was APRL VC hook, with clutch-lock disabled.

as well. With a VC device, users can control the grasp force and therefore only expend as much energy as is necessary to hold light, moderate, or heavy objects. However, the tradeoff for this increased grasp control is that the user must maintain the grip force while holding the object and hence must generate force throughout the entire task, causing fatigue, unless the device has a clutch. However, a clutch must be disengaged at the end of each movement, requiring an additional control signal, and clutches often wear out before the prehensor. VC devices accordingly seem useful for a subset of activities of daily living, but the energy tradeoff likely makes them inferior for tasks well suited for VO devices.

Several groups have designed devices that provide both VC and VO modes, either by transitioning between modes over the range of cable excursion [12-16] or by engaging a switch that transitions the device between modes [17-18]. Our group has recently developed a lightweight device with a rugged switch that may be a clinically viable solution [19]. However, no studies have objectively analyzed which tasks can be performed better using VC or VO devices. Such studies would allow prosthetists to better select an 
appropriate device based on the user's lifestyle, allow occupational therapists to give better guidance on which tasks require which type of terminal device, and provide guidance to prosthesis manufacturers on what functions and force levels should be incorporated in their designs to either optimize VO or VC devices or to balance user needs in devices that combine both modes.

The primary aim of this study was to determine whether using either a VC or a VO device makes a functional difference in activities of daily living; our longerterm goal is to assess whether providing the user with both functions (VC and VO) in one device (e.g., Sensinger et al. [19]) would improve function. Accordingly, we examined differences in functionality between clinically available VO and VC prehensors in 29 nondisabled subjects using a bypass prosthesis and 2 subjects with unilateral transradial amputations. Subjects completed the Southampton Hand Assessment Procedure (SHAP) [20] — a timed test with both abstract object manipulation and activities of daily living that is one of the few recommended research outcome measures within the field of prosthetics [21]. The SHAP consists of 26 self-timed activities through which an index of functionality (IOF)—normalized to nondisabled scores—is calculated. We measured task completion times and calculated SHAP IOF scores for both devices. Equally important, we administered a survey to assess which device type users preferred for different tasks based on their experiences with the SHAP test.

\section{MATERIALS AND METHODS}

\section{Subjects}

Twenty-nine nondisabled subjects (average age $23 \pm$ 3 yr, 17 females and 12 males, 28 right-hand dominant) and 2 subjects with a unilateral transradial amputation (ages 60 and 65 yr, both right-hand dominant men with right-side amputations) participated in this study. Both subjects with amputation had used a VO body-powered prehensor for at least 6 mo prior to testing and had nearnormal residual-limb strength. All participants gave informed written consent for this research study, which was approved by the institutional review board at Northwestern University.

\section{Bypass Socket}

An adjustable bypass prosthesis was fabricated for nondisabled subjects to wear on their dominant arm (Figure 2, left). This bypass device was controlled by a figure-of-nine harness (Figure 2, right) and had adjustable straps to accommodate a variety of wrist and forearm sizes. The prehensors incorporated a friction wrist [22] that allowed the subject to manually preposition the terminal device as desired. The bypass device used a Bowden cable to provide the same control of the prehensor available to persons with amputation using a bodypowered prosthesis. The subjects with amputation used their own socket/harness and wrist unit, with changes limited to the terminal device.

\section{Devices}

Two devices with comparable size, shape, and weight were used. Subjects were tested using the Sierra 2-Load VO hook (Hosmer Dorrance Corp; Campbell, California) (Figure 1(a)) and the APRL VC hook (Hosmer Dorrance Corp) (Figure 1(b)). Both prehensors used identical lyreshaped hooks with neoprene insets. We designed a custom piece to lengthen the palm of the Sierra 2-Load prehensor to match the length of the APRL $(16 \mathrm{~cm})$. The Sierra 2Load weighed $354 \mathrm{~g}$ and was set to $14 \mathrm{~N}$ of pinch force (the lower of the two force settings). The APRL weighed $243 \mathrm{~g}$ and the clutch lock was disabled.

\section{Southhampton Hand Assessment Procedure Test}

The SHAP test provides a reliable and objective measure of functionality [20-21]. The 26 tasks, selected from activities of daily life, require particular grasps (spherical, power, tip, tripod, lateral, or extension). The tasks are chosen to represent typical nondisabled frequency of use of these grasps. Twelve of the tasks involve moving abstract objects (light and heavy objects, 6 grasp patterns for each). The remaining 14 tasks are activities of daily living (e.g., opening a zipper or pouring water into a cup). The subject self-times each task by hitting a timer before starting and after completing the task. Recorded times are entered into the SHAP Web site, which normalizes the data, per task, based on nondisabled performance and calculates an IOF. An IOF of 100 corresponds to normal function.

Each subject was randomly assigned to start with either the VO or the VC prehensor and completed the SHAP with both prehensors. We discovered in a pilot study that subjects developed strategies for the knife-cutting and buttonboard tasks using the first prehensor (prehensor 1) such that 


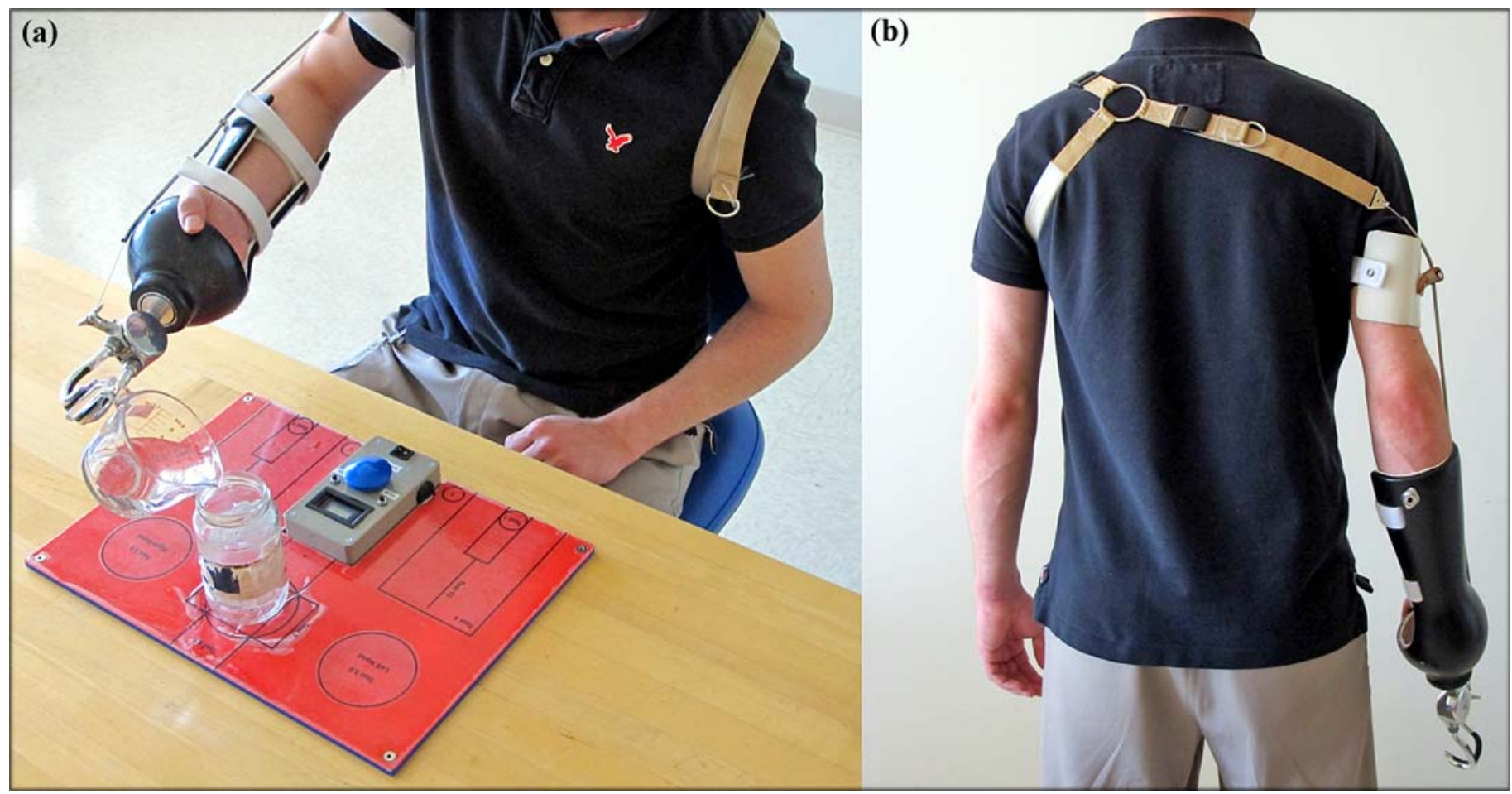

Figure 2.

(a) Photograph of experimental setup. Subjects sat in stationary chair with height adjusted so that their arm was at $90^{\circ}$ angle when resting on table. Subjects completed 26 timed tasks of Southampton Hand Assessment Procedure using each device. Pictured here is subject using voluntary opening prehensor to pour water into jar. (b) Subject wearing adjustable figure-of-nine harness.

they consistently performed better on these tasks when using prehensor 2. They afterward attributed their better performance to the strategy they developed with prehensor 1 . To eliminate this learned strategy effect, we instructed subjects to practice tasks-specifically cutting and buttoning - with both prehensors before testing. Subjects practiced 15 min with prehensor 1 , took a break, and then practiced for another 15 min with prehensor 2 .

After subjects practiced with both devices, they took the SHAP test with prehensor 1 and then prehensor 2 . Standard practices were followed with the SHAP tests including, as the SHAP protocol recommends, allowing the subject to practice each task before performing the timed test. Practicing reduced bias toward prehensor order by allowing the subject to develop comfort with the task using each prehensor before completing the timed test.

\section{Subject Survey}

After completing the SHAP tests, subjects were given a questionnaire to describe their experience with the VO and VC prehensors (Figure 3). The questionnaire had 11 open-ended questions. Questions were related to what subjects liked and disliked about each prehensor and their perceived ability to control them for different tasks.

\section{Data Processing}

For the survey, we tallied responses for which at least three subjects provided a similar response.

For nondisabled subject task completion time, we used the anovan function in MATLAB (MathWorks; Natick, Massachusetts) to perform a 4-way mixed-design analysis of variance (ANOVA) with factors of terminal device used, order in which the device was tested (to determine whether subjects routinely improved from the first to the second device tested), and two random effects: task and subject. For IOF scores, we performed a threeway ANOVA with factors of terminal device, order tested, and subject. Scores are presented as mean \pm 1 standard deviation. These analyses can determine whether one terminal device is broadly more functional than the other, but they cannot determine whether one 


\section{Survey Questions}

1. What did you like most about prehensor 1 ?

2. What did you like most about prehensor 2?

3. What did you like least about prehensor 1 ?

4. What did you like least about prehensor 2?

5. When performing a task, did you ever wish that you were wearing the other prehensor?

6. If yes to \#5, which task, and which prehensor did you wish to wear?

7. What sort of force feedback did you receive from prehensor 1? Could you feel how hard you were grasping the object?

8. What sort of force feedback did you receive from prehensor 2? Could you feel how hard you were grasping the object?

9. Did you feel like you had control over the strength of your grasp using prehensor 1 ?

10. Did you feel like you had control over the strength of your grasp using prehensor 2?

11. Would you want to use a prehensor that could switch configurations between prehensor 1 and prehensor 2?

Figure 3.

Questions listed on subject questionnaire.

device is better for some tasks. In order to determine this for a set of 26 tasks, we would have had to apply a Bonferroni adjustment factor of $\alpha=0.05 / 26$ [23]—a level of precision our pilot study did not indicate we could achieve, even with 29 subjects. Thus, the survey provides the best metric for determining which type of device should be used for each task.

In order to ensure that nondisabled subjects wearing a bypass prosthesis performed as well as experienced persons with amputation, we assessed whether data from the two subjects with amputation were consistent with those of the nondisabled subjects. We ensured that nondisabled IOF scores came from a normal distribution by using the jbtest function in MATLAB, which performs the Jarque-Bera goodness-of-fit test of composite normality [24]. We then used the Grubbs test [25] to determine whether IOF scores of persons with amputation for VC and VO devices were outliers of the nondisabled population IOF scores at an $\alpha=0.05$ level.

\section{RESULTS}

This study used the objective SHAP test and a subjective survey to evaluate what subjects liked about VO and VC devices, whether these attributes influenced which device subjects preferred for specific tasks, and whether there was an objective difference in task completion times or in the overall IOF between the two device types.

Objectively, for nondisabled subjects, task performance with the VC device (14.0 s averaged across tasks and subjects) was $1.3 \mathrm{~s}$ faster than with the VO device (15.3 s averaged across tasks and subjects, $p=0.02$ ), but this did not equate to a significantly higher IOF score $(55.4 \pm 11.5$ for VC vs $53.6 \pm 10.9$ for $\mathrm{VO}, p=0.22)$. Task times and IOF scores were not affected by the order in which subjects performed the SHAP test (i.e., with the VO device or VC device first) ( $p>0.7)$.

Both subjects with a transradial amputation conventionally used a VO device, and their VO IOF scores (45 and 71) were not significantly different from nondisabled subjects wearing the VO bypass prehensor, as determined by the Grubbs test for outliers. In contrast, of these two subjects' VC IOF scores (12 and 48), one score (12) was significantly lower than the distribution of nondisabled VC IOF scores.

Subjectively, for the VO prehensor, nondisabled subjects liked the provided pinch force (21 subjects) and specifically that they did not have to apply constant force to maintain their grasp on an object (18 subjects). Three subjects commented on the posture of the device, including that it was easy to open and place around objects, easier to 
do things closer to the body, and required minimum motion of the Bowden cable (and thus their contralateral shoulder) to operate. Many subjects also positively commented on the control of the device (7 subjects), with comments such as "better fine control," "faster," and "easy to release objects." For the VC prehensor, subjects liked aspects of the control force (25 subjects), including good grip force control (11 subjects), ability to generate high forces (8 subjects), and ability to generate low forces (6 subjects). Several subjects provided positive comments on the ability to position the VC device-specifically, the ability to position the fingers around objects since the device was open by default (4 subjects). Control was positively described (9 subjects), with comments such as "comfortable" and "natural."

When asked what they did not like about the devices, many nondisabled subjects commented on the force of the VO prehensor (20 subjects). Criticism varied from the limited grip force (10 subjects) to the excessive force for light objects ( 7 subjects), to no control over grip force (3 subjects). Three subjects had difficulty opening the fingers wide enough (although the fingers opened as wide as the VC device), and six subjects criticized aspects of control, with comments such as "difficult to maneuver" and "required more effort." For the VC prehensor, subjects disliked that they had to exert force continuously to hold objects (12 subjects). They also disliked several aspects of positioning (6 subjects), particularly holding objects in different body positions or while moving. Many subjects (9) did not like aspects of control, with comments such as "less control," "required more energy," and "had to think simultaneously about maintaining force and the location of the hook."

Subjects perceived different force feedback from the two prehensor types. Most nondisabled subjects reported that when performing tasks with the VO prehensor, they could not control (22 subjects) or perceive grip force (24 subjects). In contrast, most nondisabled subjects perceived force feedback when they performed tasks with the VC prehensor (25 subjects) and believed they could control that force (28 subjects). Both subjects with amputation reported that they could control VO force but could not control or receive feedback regarding VC force.

The majority of nondisabled subjects (27) wished at some point during a task that they had been using the other terminal device. Specifically, many subjects preferred the VO prehensor for tasks that required object rotation. These tasks included rotating a screw driver (8 subjects) and pouring water (3 subjects). Alternatively, some subjects preferred the VC prehensor for tasks that involved pouring water (7 subjects), lifting heavy objects (4 subjects), or undoing buttons ( 3 subjects).

Our survey found that if the option were available, nondisabled subjects would prefer a device that had both modes (25 subjects). Subjects most commonly explained that they would like to switch between the VO and VC prehensors to perform a variety of tasks because VO and VC prehensors each had their own strengths (25 subjects). Some subjects reported that they would like a prehensor that could switch between VO and VC prehensors only if the switch was easy to engage (4 subjects). Both subjects with amputation did not like the VC device, and accordingly did not want a device that could switch between modes.

\section{DISCUSSION}

This study used the objective, standardized SHAP test and a subjective survey to evaluate the relative strengths and weaknesses of VO and VC devices, to see whether these differences influenced when subjects wished to use them or the objective performance of the device, and to determine whether a device that could supply both modes would be advantageous.

In response to the open-ended survey questions, there was substantial consensus among subjects regarding the advantages and disadvantages of each device type that aligned well with existing clinical knowledge. The tasks for which subjects preferred each device was a logical consequence of the known attributes of that device type: for heavy objects or objects where dropping the object was nontrivial (e.g., when pouring water), many subjects preferred the higher grip strength afforded by VC devices. However, for tasks that involved simultaneous movement of joints and in which they were not concentrating solely on the prehensor (e.g., key turning) many subjects preferred the VO device, which could maintain grip force without requiring them to constantly supply it. The majority of subjects accordingly expressed a desire for a prehensor that could switch between the two modes, depending on task requirements. The preferences expressed by the subjects may also help therapists guide training when using a device that allows a change between VO and VC modes.

Using the VC device was slightly faster (1.3 s) than using the VO device. Although this difference was statistically significant, it did not result in a statistically significant 
improvement in IOF, indicating that, compared with normalized times for each of the tasks, VC did not offer a substantial improvement.

For nondisabled subjects, no difference between scores was observed based on the order in which subjects used the devices to perform the SHAP, indicating that subjects had learned to control the devices sufficiently and were comfortable performing the SHAP test. This was achieved by ensuring subjects were comfortable with performing tasks - in particular the knife-cutting and buttoning tasks in which we observed a notable learning effect in a pilot study-and allowing subjects time to explore and practice tasks before beginning the SHAP test. In addition, subjects showed no difficulty in transitioning between the two modes (e.g., practicing with device 1 , practicing with device 2 , testing with device 1 , testing with device 2). Recent studies have shown that as long as an intuitive contextual cue is provided (in this case, replacing the terminal device), subjects are able to quickly adapt back and forth between modes [26-27]. Also, the nondisabled subjects' VO IOF scores were similar to those of the subjects with amputation, who had extensive experience using a prosthesis. Given this extensive experience using VO devices in daily life, it might have been expected that they would perform better with VO devices than nondisabled subjects who had only had 15 min of practice with the device.

However, both subjects with transradial amputations had noticeably higher VO IOF scores than VC IOF scores-likely because both subjects habitually used VO prehensors on their prostheses. It was somewhat surprising that the VC IOF score of one of the subjects with amputation was significantly lower than that of nondisabled subjects. The subjects had the same limited training as the nondisabled subjects in the use of the VC device but were not able to achieve the same scores for this phase of the study. This has significant implications for therapy requirements for users in a clinical or research situation when changing control paradigms. Recent studies suggest that subjects' ability to learn a new paradigm depends on their confidence using the old paradigm: subjects can learn new paradigms more quickly if they are less confident in their old paradigms [28]. It is therefore likely that patients or subjects using one style of device who chose to then begin using both types of device, or a device that allows switching between modes, would need significant occupational therapy training-similar to the training that they received for their initial, single-mode device. Future research studies involving subjects with amputation who are already prosthetic users should ensure there is time allocated to allow for this therapy in the use of the alternative control and should also, ideally, allow for an extended trial of the device in a home environment for the most accurate comparison.

This study used one VO and one VC device. These devices were chosen because they had identical fingers; using different fingers was found to noticeably influence results in a pilot study. It is possible that different results would have been obtained if we had used different devices-for example, a VO device with a stronger or weaker pinch force, or a VC device with a clutch-lock. However, the data obtained in this study can be used as a baseline to allow comparisons between different terminal devices in future studies. The fact that subjects criticized the VO pinch force as both being too low for many tasks (10 subjects) and too high for many tasks (7 subjects) suggests that the pinch force $(14 \mathrm{~N})$ used in this study was reasonable. Since an advantage of the VC device is the ability to generate increased grip force, future studies with a device that can change between VO and VC modes would explore the optimal pinch force needed for those tasks where subjects prefer the VO mode.

In this study we only measured task completion time and IOF as objective measures of performance. It is unknown whether the VO or VC device would have performed better in other performance metrics such as cognitive burden or energy consumption. This study also only analyzed objective data comparing task speed using VO or VC devices averaged across all of the tasks_-it did not examine whether use of the VC or VO device was faster on a per-task basis. The structure of the study prevented us from analyzing the data on a per-task basis since we measured 26 tasks and, accordingly, would have had to use a substantial Bonferroni correction.

\section{CONCLUSIONS}

This study provided quantitative task completion time and IOF data for use of body-powered prostheses with both VC and VO prehensors - these data may be compared with similar data for other existing devices or new prototypes. The VC prehensor performed slightly faster across tasks than the VO prehensor, but this did not result in a statistically significant improvement in the IOF. This study also provided insights based on a questionnaire regarding which features subjects preferred, depending on the task. Subjects had consistent preferences that 
made the VO prehensor better for some tasks and the VC prehensor better for others. Subjects agreed that a device that could switch between the two modes would be advantageous. This information should also be useful both to clinicians for fitting and training patients and to engineers for designing new devices or optimizing existing ones.

\section{ACKNOWLEDGMENTS}

\author{
Author Contributions: \\ Kelsey Berning and Sarah Cohick contributed equally as co-first \\ authors. \\ Study concept and design: S. Cohick, J. W. Sensinger. \\ Acquisition of data: K. Berning. \\ Analysis and interpretation of data: K. Berning, R. Johnson, \\ J. W. Sensinger. \\ Drafting of manuscript: K. Berning, S. Cohick, R. Johnson, \\ J. W. Sensinger. \\ Critical revision of manuscript for important intellectual content: \\ L. A. Miller. \\ Statistical analysis: K. Berning, R. Johnson, J. W. Sensinger. \\ Obtained funding: J. W. Sensinger. \\ Administrative, technical, or material support: J. W. Sensinger. \\ Study supervision: J. W. Sensinger.
}

Financial Disclosures: The authors have declared that no competing interests exist.

Funding/Support: This material was based on work partially supported by the U.S. Department of Education (grant H133G120059). Initial funding of this work was generously provided by the Rice Foundation.

Additional Contributions: Dr. Sensinger is now with the Institute of Biomedical Engineering and Department of Electrical and Computer Engineering, University of New Brunswick, and the Department of Physical Medicine and Rehabilitation, Feinberg School of Medicine, Northwestern University.

Institutional Review: All participants gave informed written consent for this research study, which was approved by the institutional review board at Northwestern University.

Participant Follow-Up: The authors do not plan to inform participants of the publication of this study. However, participants have been encouraged to check the study Web site for updated publications.

Disclaimer: The contents of this article do not necessarily represent the policy of the Department of Education and endorsement of the Federal Government should not be assumed.

\section{REFERENCES}

1. Whiteside SR, Alaimo J, Barringer WJ, Beiswenger WD, Bulgarelli T, Hentges CJ, Lin RS, Miller TE, Parr RG, Reynolds JH, Stills ML. Practice analysis task force. Alexandria (VA): American Board for Certification in Orthotics and Prosthetics; 2000.
2. Weir RF, Sensinger JW. Design of artificial arms and hands for prosthetic applications. In: Kutz M, editor. Standard handbook of biomedical engineering and design. New York (NY): McGraw-Hill; 2003. p. 537-98.

3. Vodovnik L, Rebersek S. Information content of myocontrol signals for orthotic and prosthetic systems. Arch Phys Med Rehabil. 1974;55(2):52-56. [PMID:4272626]

4. Simpson DC. The choice of control system for the multimovement prosthesis: Extended physiological proprioception. In: Herberts P, editor. The control of upper-extremity prostheses and orthoses. Springfield (IL): Charles Thomas; 1974. p. 146-50.

5. Zecca M, Micera S, Carrozza MC, Dario P. Control of multifunctional prosthetic hands by processing the electromyographic signal. Crit Rev Biomed Eng. 2002;30(4-6):459-85. [PMID:12739757]

http://dx.doi.org/10.1615/CritRevBiomedEng.v30.i456.80

6. Micera S, Carpaneto J, Raspopovic S. Control of hand prostheses using peripheral information. IEEE Rev Biomed Eng. 2010;3:48-68. [PMID:22275201] http://dx.doi.org/10.1109/RBME.2010.2085429

7. McFarland LV, Winkler SL, Heinemann AW, Jones M, Esquenazi A. Unilateral upper-limb loss: Satisfaction and prosthetic-device use in veterans and servicemembers from Vietnam and OIF/OEF conflicts. J Rehabil Res Dev. 2010; 47(4):299-316. [PMID:20803400] http://dx.doi.org/10.1682/JRRD.2009.03.0027

8. Biddiss EA, Chau TT. Upper limb prosthesis use and abandonment: A survey of the last 25 years. Prosthet Orthot Int. 2007;31(3):236-57. [PMID:17979010] http://dx.doi.org/10.1080/03093640600994581

9. Pinzur MS, Angelats J, Light TR, Izuierdo R, Pluth T. Functional outcome following traumatic upper limb amputation and prosthetic limb fitting. J Hand Surg Am. 1994; 19(5):836-39. [PMID:7806814] http://dx.doi.org/10.1016/0363-5023(94)90197-X

10. Frey DD, Carlson LE, Ramaswamy V. Voluntary-opening prehensors with adjustable grip force. J Prosthet Orthot. 1995;7(4):124-31. http://dx.doi.org/10.1097/00008526-199507040-00004

11. Leblanc MA, Carlson LE. Adjustable prehension device (APD) for prosthetic hooks. Proceedings of the International Conference on Prosthetics and Orthotics; 1992; Chicago, IL. p. 67.

12. Meeks D, LeBlanc M. Preliminary assessment of three new designs of prosthetic prehensors for upper limb amputees. Prosthet Orthot Int. 1988;12(1):41-45. [PMID:3399368]

13. Procter S, LeBlanc M. Clinical Evaluation of a new design prosthetic prehensor. J Prosthet Orthot. 1991;3(2):79-83. http://dx.doi.org/10.1097/00008526-199100320-00003 
14. Kuniholm J. The open prosthetics project [Internet]. Durham (NC): The Open Prosthetics Project; 2008. Available from: http://openprosthetics.org/

15. Sensinger JW. Voluntary opening-closing terminal device design. Proceedings of the 13th International Conference on Prosthetics and Orthotics; 2010; Leipzig, Germany.

16. Leblanc MA, Parker D, Nelson C. New designs for prosthetic prehensors. Proceedings of 9th International Symposium on External Control of Human Extremities; 1987; Dubrovnik, Yugoslavia. p. 475-81.

17. Veatch BD. A combination VO/VC terminal device with variable mechanical advantage. Littleton (CO): ADA Technologies; 2004.

18. Sullivan T, Siong Teh K. Design and fabrication of a hybrid body-powered prosthetic hand with voluntary opening and voluntary closing capabilities. Proceedings of the 2011 ASME International Mechanical Engineering Congress and Exposition; 2011 Nov 11-17; Denver, CO.

19. Sensinger JW, Cohick S, Sutton L, Thomas A, Lipsey J. Voluntary-opening/voluntary-closing body-powered terminal device provides cosmesis and function. Proceedings of the 14th World Congress of the International Society for Prosthetics and Orthotics; 2013 Feb 4-7; Hyderabad, India.

20. Light CM, Chappell PH, Kyberd PJ. Establishing a standardized clinical assessment tool of pathologic and prosthetic hand function: Normative data, reliability, and validity. Arch Phys Med Rehabil. 2002;83(6):776-83. [PMID:12048655] http://dx.doi.org/10.1053/apmr.2002.32737

21. Miller LA, Swanson S. Summary and recommendations of the Academy's State of the Science conference on upper limb prosthetic outcome measures. J Prosthet Orthot. 2009;21: P83-89. http://dx.doi.org/10.1097/JPO.0b013e3181ae974d

22. Fryer CM, Stark GE, Michael JW. Body-powered components. In: Smith DG, Michael JW, Bowker JH, editors. Atlas of amputations and limb deficiencies: Surgical, prosthetic, and rehabilitation principles. 3rd ed. Rosemont (IL): American Academy of Orthopaedic Surgeons; 2004. p. 131-43.

23. Westfall PH, Young SS. Resampling-based multiple testing: Examples and methods for p-value adjustment. New York (NY): Wiley; 1993.
24. Jarque CM, Bera AK. A test for normality of observations and regression residuals. Int Stat Rev. 1987;55(2):163-72. http://dx.doi.org/10.2307/1403192

25. Grubbs FE. Procedures for detecting outlying observations in samples. Technometrics. 1969;11(1):1-21. http://dx.doi.org/10.1080/00401706.1969.10490657

26. Cothros N, Wong JD, Gribble PL. Are there distinct neural representations of object and limb dynamics? Exp Brain Res. 2006;173(4):689-97. [PMID:16525798] http://dx.doi.org/10.1007/s00221-006-0411-0

27. Cothros N, Wong J, Gribble PL. Visual cues signaling object grasp reduce interference in motor learning. J Neurophysiol. 2009;102(4):2112-20. [PMID:19657075] http://dx.doi.org/10.1152/jn.00493.2009

28. Sober SJ, Brainard MS. Vocal learning is constrained by the statistics of sensorimotor experience. Proc Natl Acad Sci U S A. 2012;109(51):21099-21103. [PMID:23213223] http://dx.doi.org/10.1073/pnas.1213622109

Submitted for publication May 28, 2013. Accepted in revised form September 19, 2013.

This article and any supplementary material should be cited as follows:

Berning K, Cohick S, Johnson R, Miller LA, Sensinger JW. Comparison of body-powered voluntary opening and voluntary closing prehensor for activities of daily life. J Rehabil Res Dev. 2014;51(2):253-62.

http://dx.doi.org/10.1682/JRRD.2013.05.0123

ResearcherID/ORCID: Sarah Cohick: C-4401-2014; Reva Johnson, MS: C-4406-2014; Jonathon W. Sensinger, PhD: C-5012-2014

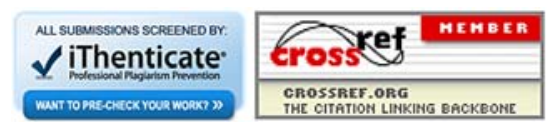


